\title{
Activation of Akt-mTOR-p70S6K pathway in angiogenesis in hepatocellular carcinoma
}

\author{
WEI LI ${ }^{1}$, DONGFENG TAN ${ }^{2}$, ZHAOPING ZHANG ${ }^{3}$, JOHN J. LIANG ${ }^{4}$ and ROBERT E. BROWN ${ }^{1}$ \\ ${ }^{1}$ Department of Pathology, University of Texas Health Science Center at Houston; ${ }^{2}$ Departments of \\ Pathology, ${ }^{3}$ Molecular Genetics U.T.M.D. Anderson Cancer, Houston, TX 77030; ${ }^{4}$ Department of Pathology, \\ Penn State Milton S. Hershey Medical Center and College of Medicine, Hershey, PA 17033, USA
}

Received April 9, 2008; Accepted May 8, 2008

\section{DOI: 10.3892/or_00000064}

\begin{abstract}
Angiogenesis is an essential process for progression of hepatocellular carcinoma (HCC). The Akt-mTOR-p70S6K signal pathway has been recognized for its roles in regulating neoangiogenesis. The role of activation of the pathway in HCC progression is poorly understood. This study aimed to evaluate the immunohistochemical expression of the phosphorylated forms of the three key constituent proteins (Akt, mTOR and p70S6K) of the Akt-mTOR-p70S6K signal pathway in HCC and non-HCC tissue. Formalin-fixed paraffin-embedded tissue sections of $51 \mathrm{HCC}, 9$ hepatocellular adenoma (HCA), 48 cirrhotic nodules $(\mathrm{CN})$ and 17 normal liver tissues (NLT) were immunostained for $\mathrm{p}-\mathrm{Akt}, \mathrm{p}-\mathrm{mTOR}$ and p-p70S6K. The number of p-Akt and p-p70S6K-positive sinusoidal endothelial cells (SEC) and the intensity of immunostaining were significantly increased in HCC compared with HA, CN and NLT $(p<0.01)$. p-mTOR in SEC tended to have an increased expression in SEC in HCC versus non-HCC tissue ( $p>0.05)$. There was a significant correlation between a high p-Akt and p-p70S6K expression, and a venous and capsular invasion of HCC. Our results suggest that activation of the Akt-mTORp70S6K pathway plays a significant role in HCC progression by promoting neoangiogenesis. Molecular strategies aimed at inhibiting this signal pathway may be of therapeutic use for the treatment of HCC.
\end{abstract}

\section{Introduction}

Hepatocellular carcinoma (HCC) is the sixth among the most common malignancies worldwide and is one of the most

Correspondence to: Dr Wei Li, Department of Pathology and Laboratory Medicine, University of Texas Health Science Center at Houston, 6431 Fannin Street, MSB 2.278 Houston, TX 77030, USA E-mail: wei.li@uth.tmc.edu

Key words: p-Akt, p-mTOR, p-p70S6K, Akt-mTOR-p70S6K pathway, hepatocellular carcinoma deadly human carcinomas because of its high recurrence and metastasis $(1,2)$. The liver contains two types of microvascular structures: large vessels (portal and central venules and hepatic arterioles) lined with continuous endothelial cells, and fenestrated sinusoids lined with discontinuous sinusoidal endothelial cells (SEC) (3). During HCC development, sinusoid fenestration is lost and capillaries are formed (sinusoidal capillarization). Modifications of the immunophenotype vascular profile consist of an abnormal distribution of endothelial markers such as CD31, CD34 and Ulex europaeus agglutinin-1, in SEC in the area of $\mathrm{HCC}(4,5)$. It has been demonstrated that angiogenesis plays a crucial role in HCC growth, invasion and metastasis (6-9). Increased interest has focused on the role of new blood vessel formation in the pathogenesis of neoplasm $(10,11)$, and the anti-angiogenic therapy of cancer types has been recognized as a promising alternative for treatment of cancer with a low toxicity and without acquisition of drug resistance because of the genetic stability and low mutational rate of endothelial cells $(12,13)$.

The mammalian target of rapamycin (mTOR), known as FKBP12 rapamycin-associated protein (FRAP/RAFT/ $\mathrm{RAPT} / \mathrm{SEP}$ ), is a $289-\mathrm{kD}$ a serine/threonine kinase (14). mTOR functions as a checkpoint for cell growth and proliferation, with upstream Akt and downstream p70S6K being the two most important mediators. Akt, also called protein kinase B (PKB), belongs to the serine/threonine protein kinase family. One of the functions of Akt is activation and phosphorylation of mTOR. Subsequently, activated mTOR regulates p70S6K activation and phosphorylation $(14,15)$. The Akt-mTORp70S6K signal pathway has been considered not only a central regulatory pathway of the protein translation involved in regulating cell proliferation, growth, differentiation and survival $(16,17)$, but also a crucial step leading to angiogenesis in the neoplastic and non-neoplastic process $(18,19)$. To our knowledge, the involvement of the Akt-mTOR-p70S6K pathway and its significance in angiogenesis in HCC has not been well documented. This study aimed to evaluate the immunohistochemical expression of the phosphorylated forms of the three key constituent proteins (p-Akt, p-mTOR and p-p70S6K) of the Akt-mTOR-p70S6K pathway in the vessels of neoplastic and non-neoplastic liver lesions, and to assess the role of this pathway in neoangiogenesis in HCC. The correlation of expression of the constituent proteins with clinicopathological factors of HCC was investigated. 

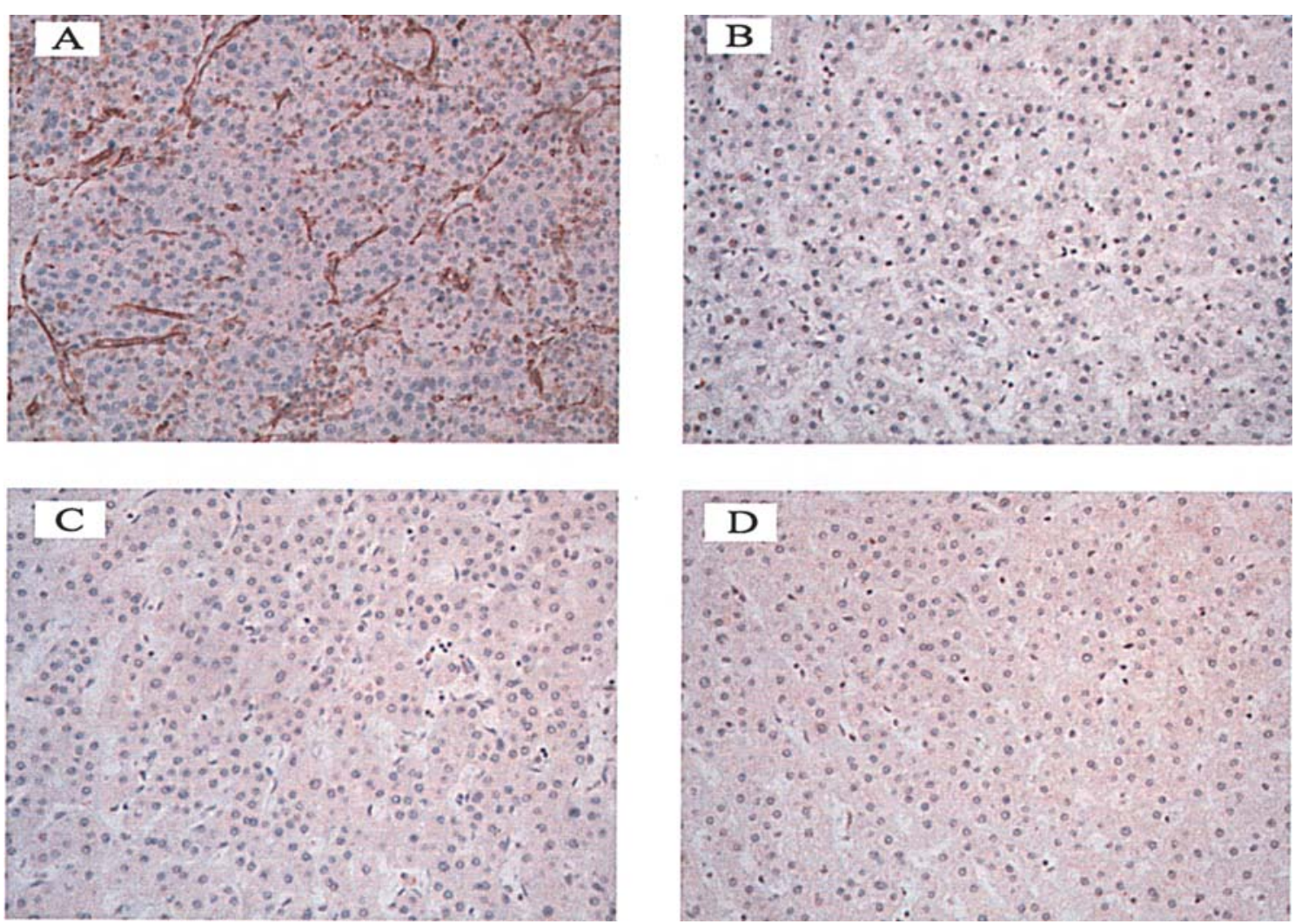

Figure 1. Expression of p-Akt in sinusoidal endothelial cells. (A) Hepatocellular carcinoma, (B) hepatocellular adenoma, (C) cirrhotic nodules and (D) normal liver tissue. Immunohistochemical staining; original magnification, x200.

Table I. Immunoreactivity scores for p-Akt.

\begin{tabular}{lccccccc}
\hline & \multicolumn{7}{c}{ Total immunoreactivity score $\mathrm{n}(\%)$} \\
\cline { 2 - 6 } & 0 & 2 & 3 & 4 & 5 & 6 & Total no. of cases \\
\hline HCC & 0 & $6(12)$ & $12(23)$ & $14(28)$ & $12(23)$ & $7(14)$ & 51 \\
HCA & $1(11)$ & $4(45)$ & $3(33)$ & $1(11)$ & 0 & 0 & 9 \\
CN & $6(13)$ & $26(54)$ & $11(23)$ & $5(10)$ & 0 & 0 & 48 \\
NLT & $3(18)$ & $12(70)$ & $1(6)$ & $1(6)$ & 0 & 0 & 17 \\
\hline
\end{tabular}

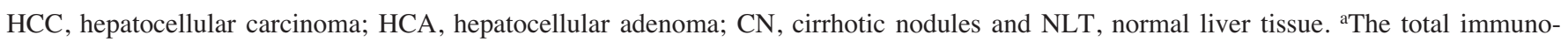
reactivity score of immunostaining was calculated by adding the percentage score (ranging from 0 to 3 ) of immunoreactive endothelial cells in the sinusoids to the intensity score (ranging from 0 to 3 ). The IRS scale does not allow the individual categories to add up to an IRS of 1.

\section{Materials and methods}

Patients and specimens. The study protocol was approved by the institute review board (IRB). The study group was composed of patients submitted to orthotopic liver transplantation or tumor resection at Memorial Hermann hospitals, affiliated hospital of the University of Texas Health Science Center at Houston. Surgical pathology cases were evaluated, including 51 HCC (14 well, 22 moderately and 15 poorly differentiated HCC) with various pathological features, 9 cases of hepatocellular adenoma and 17 normal liver tissues. Cirrhotic nodules (48 cases) were taken from non-cancerous tissue $1 \mathrm{~cm}$ away from the tumor margin. Normal liver tissue excised from traumatically injured liver (9 cases) and near hepatocellular adenoma (8 cases) was reviewed. The HCC were categorised into well, moderately or poorly differentiated types, corresponding to Edmondson's grades I/II, III or IV, respectively. The age of patients with HCC ranged from 48 to 73 years, with a mean age of 64.4. Of the 51 patients, 42 were men and 9 women. The etiology of chronic liver disease associated with HCC includes chronic hepatitis $\mathrm{C}$ virus (HCV) infection (26 cases), chronic hepatitis $\mathrm{B}$ virus (HBV) infection (3 cases), alcoholic cirrhosis (18 cases), primary biliary cirrhosis (1 case), hemochromatosis (1 case) and cryptogenetic cirrhosis (2 cases). Patients with HCA ranged in age from 26 to 48 years (mean 33 ) with a female: male ratio of $6: 1$. Tissues from the specimens were fixed in $10 \%$ buffered formalin, processed and stained with 
Table II. Immunoreactivity scores for p-p70S6K.

\begin{tabular}{|c|c|c|c|c|c|c|c|}
\hline & \multicolumn{6}{|c|}{ Total immunoreactivity score ${ }^{a} \mathrm{n}(\%)$} & \multirow[b]{2}{*}{ Total no. of cases } \\
\hline & 0 & 2 & 3 & 4 & 5 & 6 & \\
\hline $\mathrm{HCC}$ & 0 & 7 (14) & $10(20)$ & $13(25)$ & $15(29)$ & $6(12)$ & 51 \\
\hline $\mathrm{HCA}$ & $1(11)$ & $6(67)$ & $1(11)$ & $1(11)$ & 0 & 0 & 9 \\
\hline $\mathrm{CN}$ & $5(10)$ & $30(62)$ & 7 (15) & $6(13)$ & 0 & 0 & 48 \\
\hline NLT & $3(18)$ & $11(64)$ & $2(12)$ & $1(6)$ & 0 & 0 & 17 \\
\hline
\end{tabular}

HCC, hepatocellular carcinoma; HCA, hepatocellular adenoma; CN, cirrhotic nodules and NLT, normal liver tissue. ${ }^{a}$ The total immunoreactivity score of immunostaining was calculated by adding the percentage score (ranging from 0 to 3 ) of immunoreactive endothelial cells in the sinusoids to the intensity score (ranging from 0 to 3 ). The IRS scale does not allow the individual categories to add up to an IRS of 1.

hematoxylin and eosin. To confirm the diagnosis the cases were reviewed independently by two pathologists.

Immunohistochemical staining. Immunohistochemical stains were performed on formalin-fixed and paraffin-embedded $4-\mu \mathrm{m}$ sections. The tissue sections were deparaffinized in xylene and rehydrated in a graded series of ethanols. Heatinduced epitope retrieval was performed. Phospho-specific probes included antibodies directed against mTOR, p70S6K and Akt, and phosphorylated at putative sites of activation, serine (Ser) 2448, threonine (Thr) 389 and Ser 473, respectively (Cell Signaling Technology, Inc., Beverly, MA, USA). The tissue was treated with $3 \% \mathrm{H}_{2} \mathrm{O}_{2}$ and rinsed with Tween-20 (TBST) buffer. A few drops of diluted normal blocking serum were placed on the tissue and incubated at room temperature. The serum was then blotted off and the slides were incubated with primary antibody overnight at $4{ }^{\circ} \mathrm{C}$. The rest of the staining procedure was performed on a Dako autostainer programmed to treat each slide with diluted biotinylated secondary antibody for $30 \mathrm{~min}$. The slides were rinsed and incubated with DAB (3,3'-diaminobenzidine chromogen solution, EnVision+ System Kit, Dako) for $10 \mathrm{~min}$. The slides were rinsed again and counterstained with Gill II hematoxylin, treated with xylene and cover slipped. The immunohistochemical staining for CD31 (clone JC/70A, Dako Corp., Carpinteria, CA, USA) optimized at a dilution of 1:50, was performed as previously described with Dako autostainer. Positive and negative controls reacted appropriately.

Immunohistochemical staining assessment. The expression levels of the p-mTOR (Ser 2448), p-Akt (Ser473), p-p70S6K (Thr 389) and CD31 protein analytes in target cells were determined using bright-field microscopy. The staining intensity and extensiveness (percentage of cell staining) were evaluated for each antibody. A numerical scoring system with two categories was used to assess the observed expression of p-mTOR, p-Akt, p-p70S6K and CD31 in SEC. The first category documented the number of immunoreactive endothelial cells (either cytoplasmic or nuclear staining) in the sinusoids as 0 (no immunoreactive cells), $1(<10 \%), 2(10-50 \%)$ and $3(>50 \%)$. A positive case was defined as having a first category value of 1 . The second category assessed the intensity of the immunostaining (either cytoplasmic or nuclear staining) as 0 (no immunostaining), 1 (weak), 2 (moderate) and 3 (strong). Finally, the individual categories were added to give the total immunoreactivity score (IRS), which ranged from 0 to 6 . The IRS scale does not allow the individual categories to add up to an IRS of 1 . To determine the association of p-Akt, p-mTOR and p-p70 S6K with the clinicopathological parameters of HCC, we categorized the degree of staining into low expression (IRS score $\leq 4$ ) and high expression (IRS score $\geq 4$ ).

Statistical analysis. Mean values and standard deviations were calculated to describe the data population. Statistical analyses were performed using Fisher's exact test or the two-tailed t-test. $\mathrm{P}<0.05$ was considered to be significant.

\section{Results}

Expression of p-Akt in SEC. The expression of p-Akt was localized mainly in the cytoplasm of SEC. While HCA, CN and NLT showed various degrees of positive p-Akt staining in SEC, the p-Akt positive stains in SEC were observed in all cases $(100 \%)$ of HCC, and the number of immunoreactive endothelial cells and the intensity of immunostaining were significantly increased in HCC (Fig. 1A), compared with HCA, CN and NLT (Fig. 1B-D). We quantified these differences by calculating the IRS of the p-Akt positive SEC (Table I). The mean IRS and its standard deviation was $3.9 \pm 0.7$ for HCC, compared with $2.2 \pm 0.9$ for $\mathrm{HCA}, 2.2 \pm 0.8$ for $\mathrm{CN}$, and $1.8 \pm 1.1$ for NLT. The differences between the mean IRS for p-Akt were compared in pairs. $\mathrm{HCC}$ versus $\mathrm{HCA}, \mathrm{HCC}$ versus $\mathrm{CN}$, HCC versus NLT were found to be highly significant $(\mathrm{p}<0.01)$. No significant difference of p-Akt expression was observed between HCA and CN, and between HCA and NLT.

Expression of $p-p 70 S 6 K$ in SEC. Staining for p-p70S6K was predominantly localized to the cytoplasm of SEC. The immunoreactivity scores for p-p70S6K expression in SEC in HCC, HCA, CN and NLT are listed in Table II. Expression of p-p70S6K in SEC is significantly enhanced in HCC (Fig. 2A) versus non-HCC tissue (Fig. 2B-D) as regards the intensity of immunostaining and the number of immunoreactive SEC. The mean IRS and its standard deviation was 4.1 \pm 0.5 for HCC, compared with $2.1 \pm 1.0$ for HA, $2.2 \pm 0.7$ for CN and $1.9 \pm 1.0$ for NLT. The differences between the mean IRS for p-p70S6K were compared in pairs. Differences 

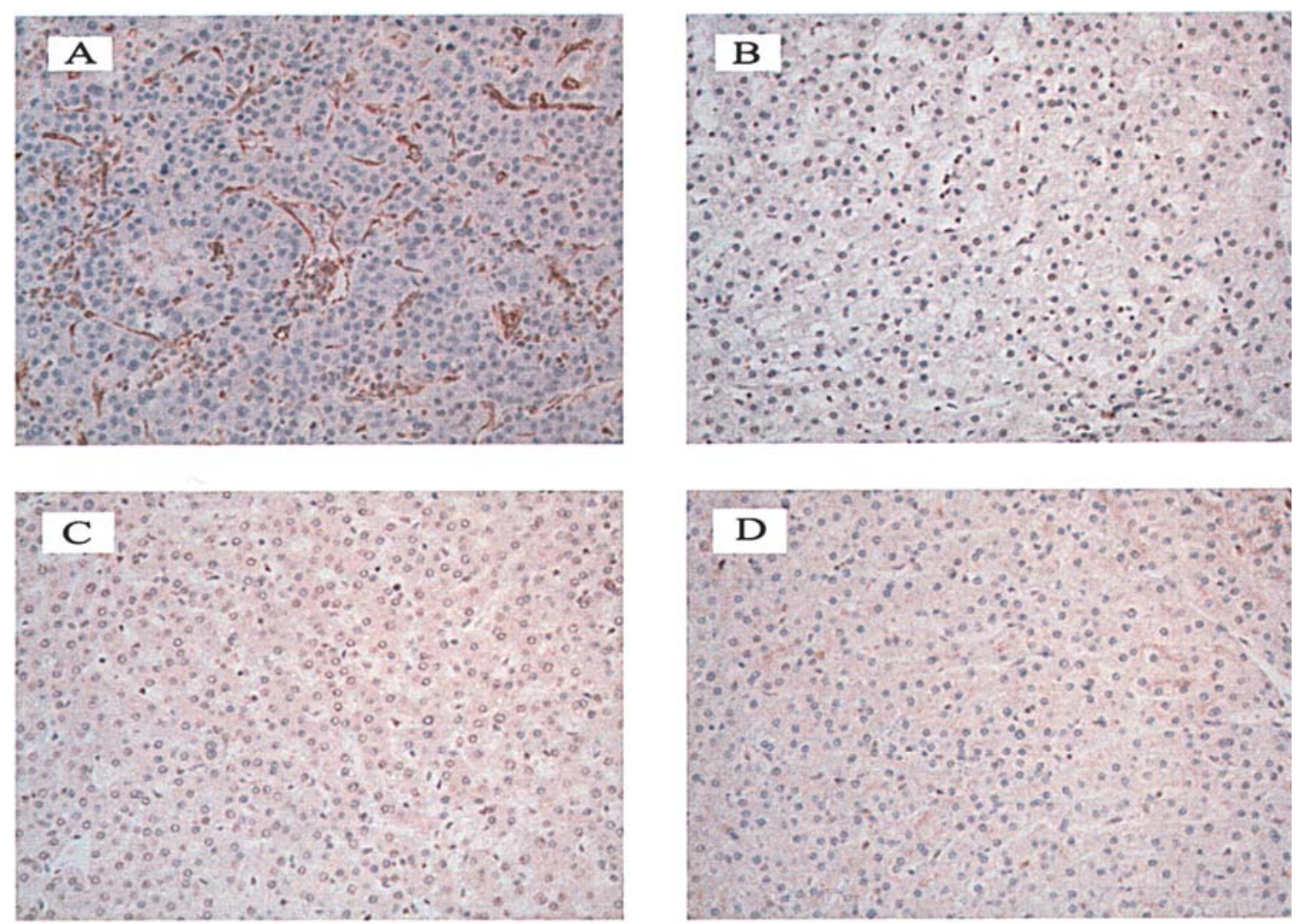

Figure 2. Expression of p-p70S6K in sinusoidal endothelial cells. (A) Hepatocellular carcinoma, (B) hepatocellular adenoma, (C) cirrhotic nodules and (D) normal liver tissue. Immunohistochemical staining; original magnification, x 200

between $\mathrm{HCC}$ and $\mathrm{HCA}, \mathrm{HCC}$ and $\mathrm{CN}, \mathrm{HCC}$ and NLT were significant $(\mathrm{p}<0.01)$. No difference of $\mathrm{p}-\mathrm{p} 70 \mathrm{~S} 6 \mathrm{~K}$ expression was observed between HCA and $\mathrm{CN}$, and between HCA and NLT.

Expression of $p$-mTOR in SEC. Staining for p-mTOR was observed in the cytoplasm/membrane and nucleus of SEC. The mean IRS and its standard deviation was $2.9 \pm 0.3$ for HCC,

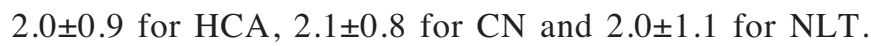
Although the expression of p-mTOR in SEC was enhanced in $\mathrm{HCC}$ versus non-HCC tissue, no significant increase was found in comparison with HCA $(\mathrm{p}=0.06), \mathrm{NC}(\mathrm{p}=0.07)$ and NLT $(p=0.08)$. No difference of $p-m T O R$ expression was observed between HCA and CN, and between HCA and NLT.

Expression of $p$-Akt, $p$-p70S6K and p-mTOR in nonsinusoidal endothelial cells, hepatocytes and other types of cells. Positive staining of p-Akt, p-p70S6K and p-mTOR in endothelial cells was found in arteries and veins of the portal tracts and fibrous septa, as well as of the terminal hepatic veins. However, no significant difference in expression was observed between HCC and non-HCC tissue. Although various degrees of p-Akt, p-p70S6K and p-mTOR expression were found in hepatocytes and fibroblasts in HCC, HCA, CN and NLT, p-Akt, p-p70S6K and p-mTOR show no significant difference in expression between HCC and non-HCC tissue. Similar levels of expression of p-Akt, p-p70S6K and p-mTOR are observed in bile duct epithelium in CN and NLT and areas adjacent to HCC and HCA. The levels and patterns of expression are similar among the groups.
Expression of the endothelial marker, CD31, was evaluated for comparison. CD31-positive sinusoids were observed in all cases $(100 \%)$ of HCC, HCA and CN, and 14 of 17 (76\%) NLT. HCC exhibited a significant increase in the expression of CD31 (mean IRS) in comparison with HCA, NC, and NLT $(\mathrm{P}<0.01)$ (data not shown). Immunostaining was mostly uniform throughout the tissue specimen.

p-Akt and p-p70S6K expression and clinicopathological features of $H C C$. We compared the clinicopathological features with groups of high (IRS score $\geq 4$ ) and low (IRS score $\leq 4)$ p-Akt and p-p70S6K expression in SEC (Table III). A high expression of $\mathrm{p}$-Akt and p-p70S6K correlated significantly with venous $(\mathrm{p}<0.001)$ and capsular invasion $(\mathrm{p}<0.01)$ of HCC. There was no significant correlation between the expression of p-Akt and p-p70S6K, and other clinicopathological variables. No significant correlation was observed between $\mathrm{p}$-mTOR expression and the clinicopathological parameter of HCC (data not shown).

\section{Discussion}

The high incidence of recurrence and metastasis has been an obstacle to improving the overall survival rate of patients with HCC. The association between angiogenesis, tumor progression and metastasis has attracted great attention. Angiogenesis is essential for any malignant tumor to grow and metastasize, including HCC. Tumor neovascularization can be established by the sprouting of blood vessels through the division of differentiated endothelial cells. There are 
Table III. Correlation between p-Akt and p-p70S6K expression and clinicopathological features of HCC.

\begin{tabular}{|c|c|c|c|c|c|c|c|}
\hline \multirow[b]{2}{*}{ Variables } & \multirow[b]{2}{*}{$\mathrm{n}$} & \multicolumn{3}{|c|}{ p-Akt expression n (\%) } & \multicolumn{3}{|c|}{ p-p70S6K expression n (\%) } \\
\hline & & $\begin{array}{l}\text { Low } \\
(\mathrm{IRS} \leq 3)\end{array}$ & $\begin{array}{l}\text { High } \\
(\mathrm{IRS} \geq 4)\end{array}$ & P-value & $\begin{array}{l}\text { Low } \\
(\mathrm{IRS} \leq 3)\end{array}$ & $\begin{array}{l}\text { High } \\
(\mathrm{IRS} \geq 4)\end{array}$ & P-value \\
\hline \multicolumn{8}{|l|}{ Age (years) } \\
\hline$\geq 60$ & 28 & $10(36)$ & $18(64)$ & NS & $9(32)$ & $19(68)$ & NS \\
\hline$<60$ & 23 & $8(35)$ & $15(65)$ & $8(35)$ & $15(65)$ & & \\
\hline \multicolumn{8}{|l|}{ Gender } \\
\hline Male & 42 & $14(33)$ & $28(69)$ & NS & $14(33)$ & $28(67)$ & NS \\
\hline Female & 9 & $4(44)$ & $5(56)$ & $3(33)$ & $6(67)$ & & \\
\hline \multicolumn{8}{|l|}{ Tumor size } \\
\hline$\geq 5 \mathrm{~cm}$ & 16 & $6(38)$ & $10(62)$ & NS & $6(38)$ & $10(62)$ & NS \\
\hline$<5 \mathrm{~cm}$ & 35 & $12(34)$ & $23(66)$ & $11(32)$ & $24(68)$ & & \\
\hline \multicolumn{8}{|c|}{ Histological grade } \\
\hline Well & 14 & $5(36)$ & $9(64)$ & NS & $5(36)$ & $9(64)$ & NS \\
\hline Moderately & 22 & $8(36)$ & $14(64)$ & $7(32)$ & $15(68)$ & & \\
\hline Poor & 15 & $5(33)$ & $10(67)$ & $5(33)$ & $10(67)$ & & \\
\hline \multicolumn{8}{|l|}{ No. of tumors } \\
\hline Solitary & 32 & $12(38)$ & $20(62)$ & NS & $10(32)$ & $22(78)$ & NS \\
\hline Multiple & 19 & $6(32)$ & $12(68)$ & 7 (37) & $12(63)$ & & \\
\hline \multicolumn{8}{|c|}{ Venous invasion } \\
\hline Yes & 10 & $1(10)$ & $9(90)$ & $<0.001$ & $0(0)$ & $19(100)$ & $<0.001$ \\
\hline No & 41 & $17(42)$ & $25(58)$ & $16(39)$ & $25(61)$ & & \\
\hline \multicolumn{8}{|c|}{ Capsular invasion } \\
\hline Yes & 34 & $5(15)$ & $29(85)$ & $<0.01$ & $6(18)$ & $28(82)$ & $<0.010$ \\
\hline No & 17 & $14(82)$ & $3(18)$ & $15(88)$ & $2(12)$ & & \\
\hline \multicolumn{8}{|c|}{ Capsular formation } \\
\hline Yes & 38 & $13(34)$ & $25(66)$ & NS & $12(32)$ & $26(68)$ & NS \\
\hline No & 13 & $5(39)$ & $8(61)$ & $5(39)$ & $8(61)$ & & \\
\hline
\end{tabular}

IRS, immunoreactivity score (see details in Tables I and II).

several ways in which the growth of new vessels can be mediated. Circulating endothelial progenitor cells derived from the bone marrow were found in the peripheral blood and implicated in the neoangiogenesis involved in tumorigenesis as well as in the formation of new vessels after trauma (20-22).

By regulating some key steps that control the balance of cell survival and apoptosis, the Akt-mTOR-p70S6K pathway has been implicated in the pathogenesis and progression of many human malignant neoplasms (23-27). However, reports on the involvement of Akt-mTOR-p70S6K pathway in the neoangiogenesis of human HCC are limited. In this study, we used immunohistochemical methods to examine expression of the phosphorylated forms of three key constituent proteins of this pathway in SEC in HCC (HCA, CN and NLT), and compare it with an endothelial marker (CD31) commonly used in surgical pathology of the liver. Our data clearly demonstrated that expression of the upstream (p-Akt) and downstream molecules (p-p70S6K) in SEC is significantly enhanced in HCC compared with HCA, CN and NLT as regards the intensity of immunostaining and the number of immunoreactive SEC. The results suggest that activation of p-Akt and p-p70S6K up-regulates angiogenesis in HCC development and progression. Expression of $p-m T O R$ in SEC appeared to be increased in HCC versus non-HCC tissue, although no significant difference was observed between the two groups. The relatively low correlation in expression between p-mTOR and its upstream effector, Akt, and between p-mTOR and its downstream effector, p-p70S6K, may raise the possibility that an alternative activation pathway may be involved besides activation of the Akt-mTOR-p70S6K pathway. Such alternatives would include those mediated by phospholipase D (PLD) via phosphatidic acid (PA), guanosine triphosphatase $\mathrm{Cdc} 42$, and lysophosphatidic acid (15,28-30), as well as the ERK1/2-p70S6K (Thr421/ Ser424) pathway (31). Further studies are needed to clarify this possibility. 
The role of activation of the serine/threonine protein kinase Akt in embryonic vascular development and neoangiogenesis has been demonstrated (32). Stimulation of the endothelium-specific receptor tyrosine kinase triggers the activation of Akt (32). Furthermore, studies have found that stimulation of the protein kinase Akt was dependent on phosphoinositide 3-OH kinase (PI3K), which mediates growth factors and hypoxia-induced Akt activation (33-35). Matsuo et al (36) reported that fibroblast growth factor-2 (FGF-2) exerted lymphangiogenic effects through the Akt/ $\mathrm{mTOR} / \mathrm{p} 70 \mathrm{~S} 6$ kinase pathway in rat lymphatic endothelial cells. They demonstrated that an obstruction of the Akt signalling pathway by LY294002 [a selective phosphatidylinositol 3-kinase (PI3K) inhibitor] abolished the elongation of tubes of lymphatic endothelial cells induced by FGF-2, while rapamycin (mTOR inhibitor) abrogated the phosphorylation of p70S6 kinase and consistently inhibited the formation of tubes of lymphatic endothelial cells induced by FGF-2 (36).

In this study, the analysis of p-Akt and p70S6K expression in relation to clinicopathological features showed that a high expression of p-Akt and p70S6K in the SEC in HCC was significantly associated with venous and capsular invasion. These data suggest an important role of activation of AktmTOR-p70S6K pathway in tumor invasiveness and progression of HCC. Venous invasion is one of the most important pathological features that lead to postoperative tumor recurrence after the resection of $\operatorname{HCC}(37,38)$. The positive association between overexpression of p-Akt, p70S6K and venous invasion further suggest the proangiogenic role of p-Akt and p-p70S6K in angiogenesis. It is well known that vascular endothelial growth factor (VEGF), the most widely studied angiogenic factor for its clinical significance, plays a significant role of VEGF in the angiogenesis of hepatocellular carcinoma (39-42). A high VEGF expression in hepatocellular carcinoma was found to not only correlate significantly with tumor staining in angiography $(39,41)$, but also be associated with portal vein tumor thrombosis (43). VEGF exerts its angiogenic actions by binding to specific plasma membrane receptors that are transmembrane glycoproteins with an extracellular ligand-binding domain and an intracellular tyrosine kinase domain (44). It has been shown that VEGF can induce PI3-kinase activity in a variety of endothelial cells, suggesting involvement of the Akt-mTORp70S6K pathway during VEGF activation (45-47).

Various levels of expression of p-Akt, p-mTOR and p70S6K were observed in hepatocytes in HCC, HCA, CN and NLT. However, these phosphorylated proteins show no significant difference in expression among the groups. Sahin et al (48) showed the overexpression of phosphorylated mTOR in hepatocytes in $15 \%$ of HCC (11 of 73 cases) and $33 \%$ in HCA (5 of 15 cases) when compared to non-neoplastic liver tissue. In their report, results of phosphorylated p70S6K expression in HCC and HCA were not reported (48).

The significance of our findings is that they identified the Akt-mTOR-p70S6K pathway as a potential target of antiangiogenetic therapy in HCC. Since angiogenesis is essential for tumor growth, metastasis and recurrence, destroying the tumor-associated microvasculature without severely damaging normal tissues is one of the ideal and alternative therapies for cancer. Rapamycin, a well-established immunosuppressive agent, has been found to possess anti-angiogenic activities $(49,50)$. Studies of its mechanism of action have shown that when bound to its intracellular receptor, the immunophilin FK506-binding protein-12, rapamycin interacts and inhibits the formation of a complex composed of mTOR, raptor and mLST8 (called mTORC1) (51). The inhibition of mTORC1 results in the hypophosphorylation of the p70S6 kinase, which is involved in regulating the translation initiation, ribosome biogenesis, and other growth and proliferation events (52). Perifosine, an Akt inhibitor, is another noteworthy therapeutic agent that targets the Akt-mTOR-p70S6K pathway. This lipid-based inhibitor inhibits the translocation of Akt to the cell membrane, and the growth of several tumors including lung, prostate, colon and melanoma in association with the inhibition of Akt activity (53). Perifosine has been found to sensitize cancer cells to apoptosis and cell cycle arrest induced by radiation (54). It will be of note to see whether perifosine has any anti-angiogenetic effect.

In conclusion, our study provides evidence that $\mathrm{p}$-Akt, p-mTOR and p-p70S6K are detected in SEC in HCC tissue, and that the expression of p-Akt and p-p70S6K is significantly higher than in non-HCC tissues. Our results suggest that the Akt-mTOR-p70S6K pathway is involved in HCC progression by promoting neoangiogenesis, suggesting possible novel targets for therapeutic intervention to prevent HCC progression. The molecular mechanism(s) of activation of the Akt-mTORp70S6K pathway in HCC progression merits further investigation.

\section{References}

1. Tang ZY: Hepatocellular carcinoma. J Gastroenterol Hepatol 15: 1-7, 2000.

2. Fan ST, Lo CM, Liu CL, et al: Hepatectomy for hepatocellular carcinoma: toward zero hospital death. Ann Surg 229: 322-330, 1999.

3. Folkman $\mathrm{J}$ : What is evidence that tumors are angiogenesis dependent? J Natl Cancer Invest 82: 4-6, 1990.

4. McCuskey RS and Reilly F: Hepatic microvasculature: dynamic structure and its regulation. Sem Liver Dis 12: 1-12, 1993.

5. Ruck P, Xiao JC and Kaiserling E: Immunoreactivity of sinusoids in hepatocellular carcinoma. An immunohistochemical study using lectin UEA-1 and antibodies against endothelial markers, including CD34. Arch Pathol Lab Med 119: 173-178, 1995.

6. Cui S, Hano H, Sakata A, et al: Enhanced CD34 expression of sinusoid-like vascular endothelial cells in hepatocellular carcinoma. Pathol Int 46: 751-756, 1996.

7. Poon RT, Ng IO, Lau C, et al: Tumor microvessel density as a predictor of recurrence after resection of hepatocellular carcinoma: a prospective study. J Clin Oncol 20: 1775-1785, 2002.

8. Tanigawa N, Lu C, Mitsui T and Miura S: Quantitation of sinusoid-like vessels in hepatocellular carcinoma: its clinical and prognostic significance. Hepatology 26: 1216-1223, 1997.

9. Sugimachi K, Tanaka S, Terashi T, Taguchi K, Rikimaru T and Sugimachi K: The mechanisms of angiogenesis in hepatocellular carcinoma: angiogenic switch during tumor progression. Surgery 131: 135-141, 2002.

10. Folkman J: Anti-angiogenesis: new concept for therapy of solid tumors. Ann Surgery 175: 409-416, 1972.

11. Folkman J: Tumor angiogenesis: therapeutic implications. J Natl Cancer Inst 285: 1182-1186, 1971.

12. Carmeliet P and Jain RK: Angiogenesis in cancer and other diseases. Nature 407: 249-257, 2000.

13. Boehm T, Folkman J, Browder T and O'Reilly MS: Antiangiogenic therapy of experimental cancer does not induce acquired drug resistance. Nature 390: 404-407, 1997.

14. Gingras AC, Raught B and Sonenberg N: Regulation of translation initiation by FRAP/mTOR. Genes Dev 15: 807-826, 2001 . 
15. Chen J and Fang Y: A novel pathway regulating the mammalian target of rapamycin (mTOR) signaling. Biochem Pharmacol 64 1071-1077, 2002

16. Pyronnet S and Sonenberg N: Cell-cycle-dependent translational control. Curr Opin Genet Dev 11: 13-18, 2001.

17. Schmelzle T and Hall MN: TOR: a central controller of cell growth. Cell 103: 253-262, 2000.

18. Maffucci T, Piccolo E and Cumashi A, et al: Inhibition of the phosphatidylinositol 3-kinase/Akt pathway by inositol pentakisphosphate results in antiangiogenic and antitumor effects Cancer Res 65: 8339-8349, 2005.

19. Shiojima I and Walsh K: Role of Akt signaling in vascular homeostasis and angiogenesis. Circ Res 90: 1243-1250, 2002.

20. Asahara T, Masuda H, Takahashi T, et al: Bone marrow origin of endothelial progenitor cells responsible for postnatal vasculogenesis in physiological and pathological neovascularization. Circ Res 85: 221-228, 1999.

21. Lyden D, Hattori K, Dias S, et al: Impaired recruitment of bonemarrow-derived endothelial and hematopoietic precursor cells blocks tumor angiogenesis and growth. Nat Med 7: 1194-1201, 2001.

22. Gill M, Dias S, Hattori K, et al: Vascular trauma induces rapid but transient mobilization of VEGFR2 (+)AC133(+) endothelial precursor cells. Circ Res 88: 167-174, 2001.

23. McCarty M: Targeting multiple signaling pathways as a strategy for managing prostate cancer: multifocal signal modulation therapy. Integr Cancer Ther 3: 349-380, 2004.

24. David O, Jett J, LeBeau H, Dy G, Hughes J, Friedman M and Brody AR: Phospho-Akt overexpression in non-small cell lung cancer confers significant stage-independent survival disadvantage. Clin Cancer Res 10: 6865-6871, 2004

25. Miyakawa M, Tsushima T, Murakami H, Wakai K, Isozaki O and Takano K: Increased expression of phosphorylated p70S6 kinase and Akt in papillary thyroid cancer tissues. Endocr J 50: 77-83, 2003.

26. Zhou X, Tan M, Stone Hawthorne V, et al: Activation of Akt/ mammalian target of rapamycin/4E BP1 pathway by ErbB2 overexpression predicts tumor progression in breast cancers. Clin Cancer Res 10: 6779-6788, 2004.

27. Lin F, Zhang PL, Yang XJ and Brown RE: Morphoproteomic and molecular concomitants of an overexpressed and activated mTOR pathway in renal cell carcinomas. Ann Clin Lab Sci 36: 283-293, 2006

28. Chen Y, Rodrik V and Foster DA: Alternative phospholipase $\mathrm{D} / \mathrm{mTOR}$ survival signal in human breast cancer cells. Oncogene 20: 672-679, 2005.

29. Chen Y, Zheng Y and Foster DA: Phospholipase D confers rapamycin resistance in human breast cancer cells. Oncogene 22: 3937-3942, 2003

30. Fang Y, Park IH, Wu AL, et al: PLD1 regulates mTOR signaling and mediates Cdc42 activation of S6K1. Curr Biol 13: 2037-2044, 2003.

31. Page G, Khidir FA, Pain S, et al: Group I metabotropic glutamate receptors activate the p70S6 kinase via both mammalian target of rapamycin (mTOR) and extracellular signal-regulated kinase (ERK 1/2) signaling pathways in rat striatal and hippocampal synaptoneurosomes. Neurochem Int 49: 413-421, 2006.

32. Kontos CD, Stauffer TP, Yang WP, et al: Tyrosine 1101 of Tie 2 is the major site of association of $\mathrm{p} 85$ and is required for activation of phosphatidylinositol 3-kinase and Akt. Mol Cell Biol 178: 4131-4140, 1998 .

33. Datta K, Bellacosa A, Chan TO and Tsichlis PN: Akt is a direct target of the phosphatidylinositol 3-kinase: activation by growth factors, v-src and v-Ha-ras, in Sf9 and mammalian cells. J Biol Chem 271: 30835-30839, 1996.

34. Stephens L, Anderson K, Stokoe D, et al: Protein kinase B kinases that mediate phosphatidylinositol 3,4,5-triphosphate-dependent activation of protein kinase B. Science 279: 710-714, 1998.
35. Downward J: Lipid-regulating kinases: some common themes at last. Science 279: 673-674, 1998.

36. Matsuo M, Yamada S, Koizumi K, Sakurai H and Saiki I: Tumour-derived fibroblast growth factor-2 exerts lymphangiogenic effects through Akt/mTOR/p70S6kinase pathway in rat lymphatic endothelial cells. Eur J Cancer 43: 1748-1754, 2007.

37. Poon RT, Fan ST and Wong J: Risk factors, prevention, and management of postoperative recurrence after resection of hepatocellular carcinoma. Ann Surg 232: 10-24, 2001.

38. Poon RT: Angiogenesis in hepatocellular carcinoma. Asian J Surg 23: 259-267, 2000.

39. Mise M, Arii S, Higashituji H, et al: Clinical significance of vascular endothelial growth factor and basic fibroblast growth factor gene expression in liver tumor. Hepatology 23: 455-464, 1996.

40. Miura H, Miyazaki T, Kuroda M, et al: Increased expression of vascular endothelial growth factor in human hepatocellular carcinoma. J Hepatol 27: 854-861, 1997

41. Gorrin-Rivas MJ, Arii S, Mori A, et al: Implications of human macrophage metalloelastase and vascular endothelial growth factor gene expression in angiogenesis of hepatocellular carcinoma. Ann Surg 231: 67-73, 2000

42. Kwak BK, Shim HJ, Park ES, et al: Hepatocellular carcinoma: correlation between vascular endothelial growth factor level and degree of enhancement by multiphase contrast-enhanced computed tomography. Invest Radiol 36: 487-492, 2001.

43. Zhou J, Tang ZY and Fan J, et al: Expression of platelet-derived endothelial cell growth factor and vascular endothelial growth factor in hepatocellular carcinoma and portal vein tumor thrombus. J Cancer Res Clin Oncol 126: 57-61, 2000.

44. Ferrara, N, Houck KA, Jakeman LB and Leung DW: Molecular and biological properties of the vascular endothelial growth factor family of proteins. Endocr Rev 13: 18-32, 1992.

45. Guo D, Jia Q, Song HY, Warren RS and Donner DB: Vascular endothelial cell growth factor promotes tyrosine phosphorylation of mediators of signal transduction that contain $\mathrm{SH} 2$ domains. Association with endothelial cell proliferation. J Biol Chem 270: 6729-6733, 1995.

46. Xia P, Aiello LP, Ishii $\mathrm{H}$, et al: Characterization of vascular endothelial growth factor's effect on the activation of protein kinase C, its isoforms, and endothelial cell growth. J Clin Invest 98: 2018-2026, 1996

47. Kevil CG, Payne DK, Mire E and Alexander JS: Vascular permeability factor/vascular endothelial cell growth factormediated permeability occurs through disorganization of endothelial junctional proteins. J Biol Chem 273: 15099-15103, 1998

48. Sahin F, Kannangai R, Adegbola O, Wang J, Su G and Torbenson M: mTOR and P70 S6 kinase expression in primary liver neoplasms. Clin Cancer Res 10: 8421-8425, 2004.

49. Vignot S, Faivre S, Aguirre D and Raymond E: mTOR-targeted therapy of cancer with rapamycin derivatives. Ann Oncol 16: 525-537, 2005

50. Sawyers CL: Will mTOR inhibitors make it as cancer drugs? Cancer Cell 4: 343-348, 2003.

51. Chiu MI, Katz H and BerlinV: RAPT1, a mammalian homolog of yeast Tor, interacts with the FKBP12/ rapamycin complex. Proc Natl Acad Sci USA 91: 12574-12578, 1994.

52. Burnett PE, Barrow RK, Cohen NA, Snyder SH and Sabatini DM: RAFT1 phosphorylation of the translational regulators p70 S6 kinase and 4E-BP1. Proc Natl Acad Sci USA 95: 1432-1437, 1998.

53. Crul M, Rosing H, de Klerk GJ, et al: Phase I and pharmacological study of daily oral administration of perifosine (D-21266) in patients with advanced solid tumours. Eur J Cancer 38: 1615-1621, 2002.

54. Vink SR, Lagerwerf S, Mesman E, et al: Radiosensitization of squamous cell carcinoma by the alkylphospholipid perifosine in cell culture and xenografts. Clin Cancer Res 12: 1615-1622, 2006. 